\title{
A Neural Network and Web-Based Decision Support System for Forex Forecasting and Trading
}

\author{
K.K. Lai ${ }^{1}$, Lean $\mathrm{Yu}^{2,3}$, and Shouyang Wang ${ }^{2,4}$ \\ ${ }^{1}$ Department of Management Sciences, City University of Hong Kong, \\ Tat Chee Avenue, Kowloon, Hong Kong \\ mskklai@cityu.edu.hk \\ ${ }^{2}$ Institute of Systems Science, Academy of Mathematics and Systems Sciences, \\ Chinese Academy of Sciences, Beijing 100080, China \\ \{yulean, sywang\}@mail.amss.ac.cn \\ ${ }^{3}$ School of Management, Graduate School of Chinese Academy of Sciences, \\ Chinese Academy of Sciences, Beijing 100039, China \\ ${ }^{4}$ Institute of Policy and Planning Sciences, University of Tsukuba, \\ Tsukuba, Ibaraki 305-8573, Japan \\ wang@sk.tsukuba.ac.jp
}

\begin{abstract}
This study presents a neural network \& web-based decision support system (DSS) for foreign exchange (forex) forecasting and trading decision, which is adaptable to the needs of financial organizations and individual investors. In this study, we integrate the back-propagation neural network (BPNN)based forex rolling forecasting system to accurately predict the change in direction of daily exchange rates, and the Web-based forex trading decision support system to obtain forecasting data and provide some investment decision suggestions for financial practitioners. This research reveals the structure of the DSS by the description of an integrated framework, and meantime we find that the DSS is integrated, user-oriented by its implementation, and practical applications reveal that this DSS demonstrates very high forecasting accuracy and its trading recommendations are reliable.
\end{abstract}

\section{Introduction}

Decision support system (DSS) is a powerful tool which can be used to support decision makers in making strategic decisions [1]. One of their objectives is to improve management judgment by fostering understanding and insights and by allowing appropriate access to relevant information [2]. The ability to forecast the future, based only on past data, leads to strategic advantages, which may be the key to success in organizations. In real life, one would be interested not only in efforts in forecasting, but also in practical trading strategies with possibility of taking positions in the foreign exchange (forex) market. Furthermore, Tsoi et al. in their earlier studies have shown that the direction of the forecast is more important than the actual forecast itself in determining the profitability of a forecasting and trading system [3]. In addition, the forex market is a rather complicated environment, and good predictions for 
its developments are key to successful trading. Traders must predict forex price movements in order to sell at top range and to buy at bottom range. Forecasting often plays an important role in the process of decision-making. Hence, forecasting system is always connected with the DSS to improve decision-making.

However, there is currently no integrated DSS approach that combines the ANNbased forecasting and web-based decision implementation to support predicting and trading decision in forex markets. We therefore propose an integrated framework approach that can implement the forex forecasting and present trading strategies in forex trading. Our approach, based on the framework, is as follows: first, the BPNNbased forex rolling forecasting system (BPNNFRFS) provides the prediction results of main international currencies employing burgeoning artificial intelligence technology to forex trading decision support system; second, the web-based forex trading decision support system (WFTDSS) is developed to provide some trading signals or trading suggestions for investors according to some corresponding decision criteria. In addition, the BPNNFRFS is constructed using artificial neural networks, while the WFTDSS uses various decision techniques and web technologies. Moreover, the integrated forex rolling forecasting and trading decision support system (IFRFTDSS), which integrates BPNNFRFS and WFTDSS, is designed to provide investors with daily trading suggestions on the forex markets. In the following section, we start with a brief introduction to the integrated framework of IFRFTDSS. Then we describe how a BPNNFRFS and a WFTDSS can be built around the framework. We also show how to incorporate two individual systems into an integrated DSS with support for financial institutions and individual investors. At the same time, we provide a brief description of IFRFTDSS, and show the implementations of the IFRFTDSS. Finally, some conclusions are made.

\section{The Proposed Framework for IFRFTDSS}

This section first describes the general integrated framework of IFRFTDSS, and discusses the two subsystems - BPNNFRFS and WFTDSS - which will be employed in the proposed integrated framework. The integrated system in the framework, IFRFTDSS, will be described in next section.

\subsection{The General Integrated Framework}

The integrated framework of IFRFTDSS proposed in this study will be developed to support decision making in forex trading with the use of the forex rolling forecasting results and various trading decision criteria. That is, it is designed to generate sell/buy/hold signals to support forex trading decisions. Based on this framework, we develop an IFRFTDSS for main international currencies. IFRFTDSS consists of two subsystems: one is the BPNN-based Forex rolling forecasting system (BPNNFRFS), which is the off-line training and forecasting unit; the other is the web-based forex trading decision support system (WFTDSS), which is the daily on-line prediction and decision unit. The former can provide database support for the latter. That is to say, 
they couple with the data that is produced by the BPNNFRFS. The general framework we propose is shown in Fig. 1.

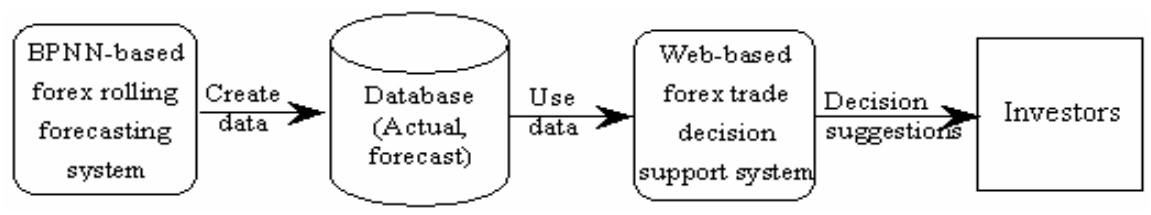

Fig. 1. The general integrated framework

\subsection{The BPNN-Based Forex Rolling Forecasting System - BPNNFRFS}

Studies [1, 3, 4, 5, 6-9] reveal that there are many applications for forex forecasting with BPNN, but it is hard to find a whole forecasting system based on BPNN techniques in the literature. Furthermore, most existing forecasting methods and tools do not have good interaction or flexibility, as the practitioners are required to have a thorough theory background in order to understand the methodologies and models. Hence, it is difficult to achieve good decision alternatives based on these models and tools. Although methods and tools used in their works are quite diverse, satisfactory forecasting results have not been obtained so far. Thus, this study aims to develop a whole forex forecasting system to forecast a change in direction or trend in forex movements for some main international currencies employing burgeoning artificial intelligence technology. The rolling forecasting technique is also introduced to improve forecasting accuracy in this forecasting system. Thus, the BPNN-based Forex rolling forecasting system - BPNNFRFS - is generated. The main user interface of the BPNNFRFS is shown in Fig. 2.

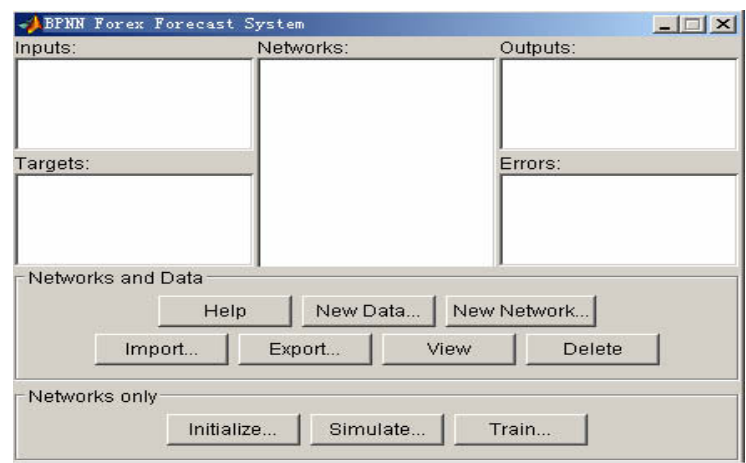

Fig. 2. The user interface of BPNNFRFS

The BPNNFRFS is built using the Matlab software, which is produced by Mathworks Laboratory Corporation. The main flows in this system contain eight key stages, i.e., sample selection, data division, data preprocessing, determination of net- 
work architecture, initialization, sample training, sample validation, and simulation and forecast, as shown in Fig. 3.

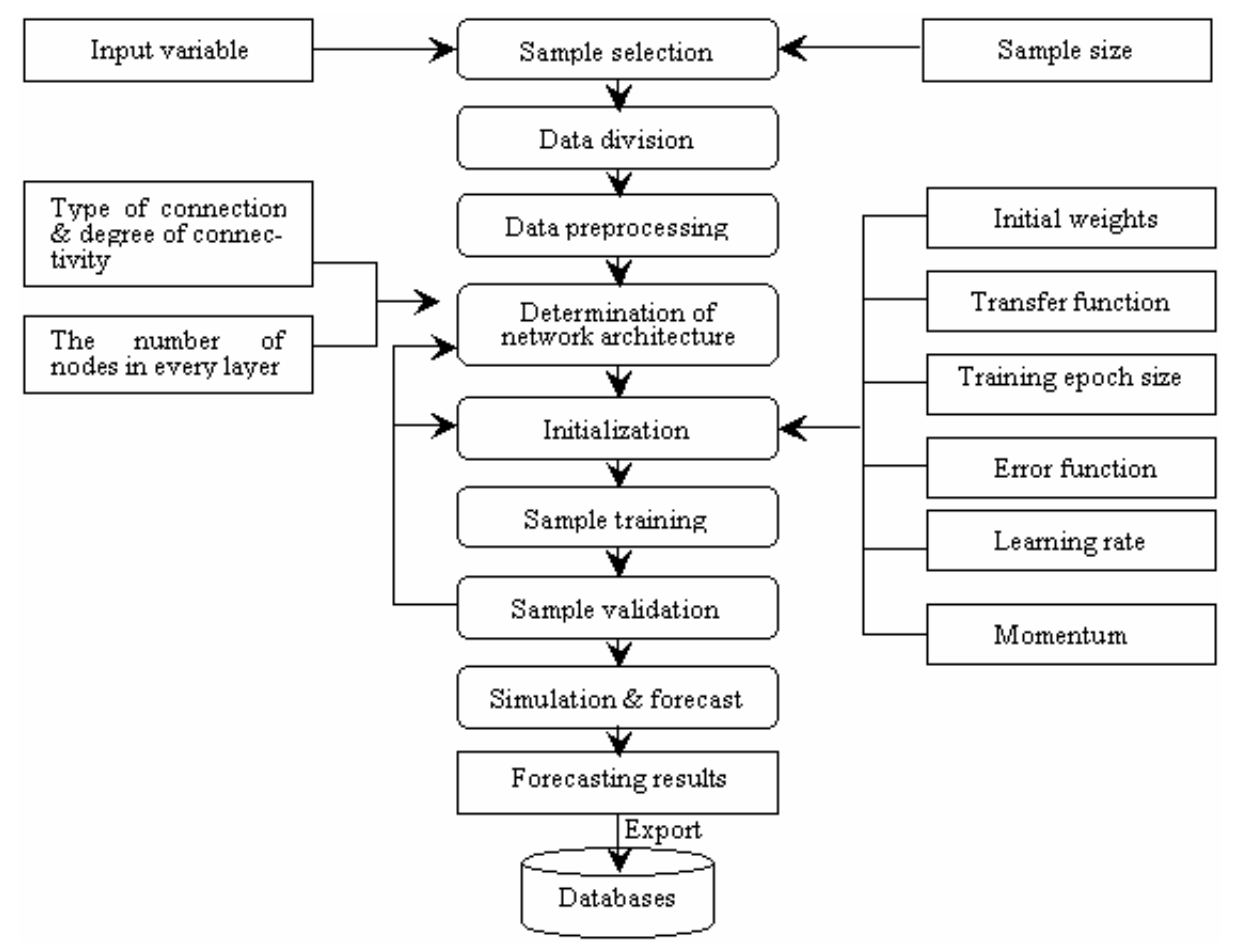

Fig. 3. The BPNNFRFS flow chart

From Fig.3, we can see that this is a relatively complex process. However, easy operating is an important measurement of software system. In order to facilitate forecasters and investors, we develop this easy-use subsystem (see Fig.2) for forex prediction. Especially, this system can be used independently as a forecasting system.

In order to improve prediction accuracy and performance, rolling forecasting techniques are introduced into this subsystem. Rolling forecasting techniques with neural networks are referred to [8].

\subsection{The Web-Based Forex Trading Decision Support System - WFTDSS}

WFTDSS is developed to support decision making in forex trading. It is designed to convert forex price information that is presented by BPNNFRFS into forex movement tendency information, thus generating sell/buy/hold signals directly for investors. In this system, four trading rules are presented for users' choice. Different trading rules may result in different trading strategies. In order to introduce these judgment rules, the forex actual and predicted values at time $t, x_{t}$ and $\hat{x}_{t}$, are defined here. 
The first rule is a simple price comparison judgment criterion. In general, this criterion is the simplest of all, and includes the following three rules.

Rule I: If $\left(\hat{x}_{t+1}-x_{t}\right)>0$, then the forex price trend is "upward" and the current trading strategy is "buy".

Rule II: If $\left(\hat{x}_{t+1}-x_{t}\right)<0$, then the forex price trend is "downward" and the current trading strategy is "sell".

Rule III: If $\left(\hat{x}_{t+1}-x_{t}\right)=0$, then forex price is "unchangeable" and the current trading strategy is "hold and deposit".

It is worth noting that use of this rule has no man's participation (i.e., parameter specification), but the forex trading strategies can still be generated automatically.

The second rule is a cost-adjusted filter judgment criterion. This rule introduces cost as adjusted bands. The objective of the band is to reduce the number of buy (sell) signals by eliminating weak signals when the predicted value $\hat{x}_{t+1}$ and the actual value $x_{t}$ are very close. After considering funding costs and transaction costs, some trading is not worth executing. Thus, this rule can help a trading system eliminate unnecessary trading and gain more profits. Mathematically the trading rules in their simplest form can be expressed as follows.

Rule I: If $\left(\hat{x}_{t+1}-x_{t}\right)>c$, then the current trading strategy is " $b u y$ ".

Rule II: If $\left(\hat{x}_{t+1}-x_{t}\right)<c$, then the current trading strategy is "sell".

Rule III: If $\left(\hat{x}_{t+1}-x_{t}\right)=c$, then the current trading strategy is "hold and deposit".

where $c$ denotes cost, including all transaction costs and funding costs.

The third rule is a probability-based threshold judgment criterion. The trading probability based on the predicted forex price return is the basis of this trading judgment criterion. Its procedures include three steps as follows.

Firstly, let the forex price and forex price return at day $t$ be denoted by $x_{t}$, and $R_{t}$. Based on the predicted value $\hat{x}_{t+1}$ that is produced by BPNNFRFS, the forex price return can also be calculated by

$$
\hat{R}_{t+1}=\left(\hat{x}_{t+1}-x_{t}\right) / x_{t}
$$

Secondly, let the "buy" and "sell" probability of the forex price return be denoted by $B_{t+1(j)}, S_{t+1(j)}$, respectively. As the forex price is a stochastic process, the probability $B_{t+1(j)}$ and $S_{t+1(j)}$ for the next day are calculated, respectively, by

$$
\begin{aligned}
& B_{t+1(j)}=P\left\{R_{t+1(j)}>0\right\} \quad(j=1,2, \ldots, N) \\
& S_{t+1(j)}=P\left\{R_{t+1(j)}<0\right\} \quad(j=1,2, \ldots, N)
\end{aligned}
$$

where $j$ denotes the number of forex candidates.

In the "buy" case, the basic rule is that the predicted forex price of the next day is higher than the current price, i.e. the predicted forex price return is larger than zero. In the "sell" case, the basic criterion is that the predicted forex price of the next day is 
lower than the current price, i.e. the predicted forex price return should be smaller than zero. It is worth noting that in the "buy" case, the forex with the largest trading probability $B_{t+1(\max )}$ is chosen from the trading probability $B_{t+1(j)}$ of all $N$ forex candidates by

$$
B_{t+1(\max )}=\max \left\{B_{t+1(1)}, B_{t+1(2)}, \ldots, B_{T+1(N)}\right\}
$$

Thirdly, the thresholds for buying and selling, $\theta_{B}$ and $\theta_{S}$, are set in advance.

Up until now, the corresponding trading judgment rules are given by:

Rule I: If $B_{t+1(\max )} \geq \theta_{B}$, then the trading strategy is " $b u y$ ".

Rule II: If $S_{t+1(j)} \geq \theta_{S}$, then the trading strategy is "sell".

In this criterion, once the users or decision-makers specify a certain threshold, the optimal trading strategies will be presented explicitly.

The fourth criterion is risk-adjusted forex trading judgment criterion, which originated from the work of Chen and Leung [9]. Their work assumed that the forex market investment returns are determined by three features: forex appreciation, forex depreciation, and interest received from the money market. Let $r$ and $r^{*}$ be the daily domestic and foreign money market rates, and $x_{t}$ and $\hat{x}_{t+1}$ be the observed exchange rates at day $t$ and the forecast of exchange rate at day $(t+1)$ respectively. Given the notion that an investor is risk averse and can earn risk free interest from the domestic money market, we need to discount the expected rate of return based on a long or a short position by a risk aversion factor, $\gamma$. The risk aversion factor $\gamma$ can take on any real value greater than negative one $(\gamma>-1.0)$. For the risk neutral case, $\gamma$ is zero $(\gamma=0.0)$. The value of $\gamma$ is greater than zero $(\gamma>0.0)$ if the investor is risk averse. On the other hand, the value of $\gamma$ is between zero and negative one $(-1.0<\gamma<0.0)$ if the investor is a risk lover.

Based on this logical framework, we develop a risk-adjusted forex trading rule. The detailed expressions are as follows (for derivation of these conditions, refer to [9]).

Rule I: If $\ln \left(\frac{\hat{x}_{t+1}}{x_{t}}\right)<\left(r^{*}-r\right)(1+\gamma)$ and $\left(r^{*}-r\right)(1+\gamma)<0$, then trading strategy is "buy".

Rule II: If $\ln \left(\frac{\hat{x}_{t+1}}{x_{t}}\right) \leq \frac{\left(r^{*}-r\right)(1+\gamma)}{2}$ and $\left(r^{*}-r\right)(1+\gamma) \geq 0$, then the strategy is "buy".

Rule III: If $\ln \left(\frac{\hat{x}_{t+1}}{x_{t}}\right)>\frac{\left(r^{*}-r\right)(1+\gamma)}{2}$ and $\ln \left(\frac{\hat{x}_{t+1}}{x_{t}}\right)>0$, then the trading strategy is "sell".

Rule IV: If $\ln \left(\frac{\hat{x}_{t+1}}{x_{t}}\right) \geq\left(r^{*}-r\right)(1+\gamma)$ and $\ln \left(\frac{\hat{x}_{t+1}}{x_{t}}\right) \leq 0$, then the trading strategy is "hold and deposit".

Compared with the previous three criteria, this criterion seems to be relative complex. However, once the values of $r^{*}, r$ and $\gamma$ are specified by users or decision- 
makers, the optimal trading strategies will also be generated automatically regardless of paying attention to meaning of optimal conditions.

Of course, we can also implement more complex pre-defined or user-defined conditions and more complex judgment rules, and thus more optimal trading criteria and trading strategies will be generated. In our DSS, the knowledge base (KB) is formed by these rules and other judgment criteria. Besides these rules and criteria, there are many heuristic methodologies that deal with univariate time series data, e.g. how to convert univariate forex time series into input matrix vector for BPNN training, the allocation size in each traded currency. Thus the model base (MB) is generated by these models.

Based on the $\mathrm{KB}$ and $\mathrm{MB}$, plus the database (DB) already produced by the BPNNFRFS, the web-based forex trading decision support system (WFTDSS) is constructed with the aid of popular web technology. Fig. 4 shows the basic architecture of this subsystem and the data flow between user interface, KB, MB and DB. Basically, this system contains the following components.

User-Friendly Interface. Used by decision-makers and investors to input some data and query conditions, and to select some trading decision methods and retrieve answers from related services. It also displays to the users and allows them to interact with the system to arrive at satisfactory solutions.

$M B$. Used to handle models of the many different types, which may be chosen.

$K B$. Used to judge the forex change tendency and determine forex trading decisions.

$D B$. Used as a repository of historical data and forecasting data for all model programs. In the meantime, it provides data support for the KB and MB needed.

Security System. Not only used for user protection purposes, but also checks for user access levels to determine the level of flexibility and interaction that the system would allow or provide.

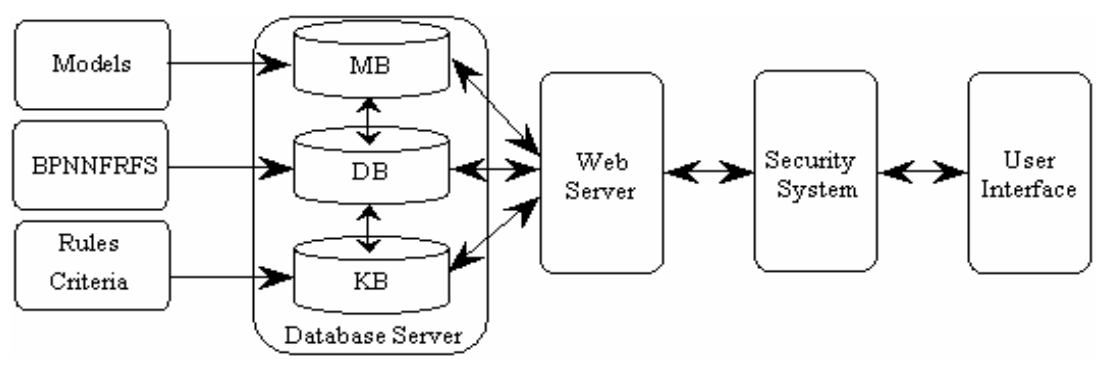

Fig. 4. The basic architecture and data flow of the WFTDSS

According to the previous WFTDSS architecture and data flow, we develop the web-based forex trading decision system adopting popular web technology and using the browser/server mode, which contains a three-tier structure. The basic construction of three-tier structure is shown in Fig. 5. 


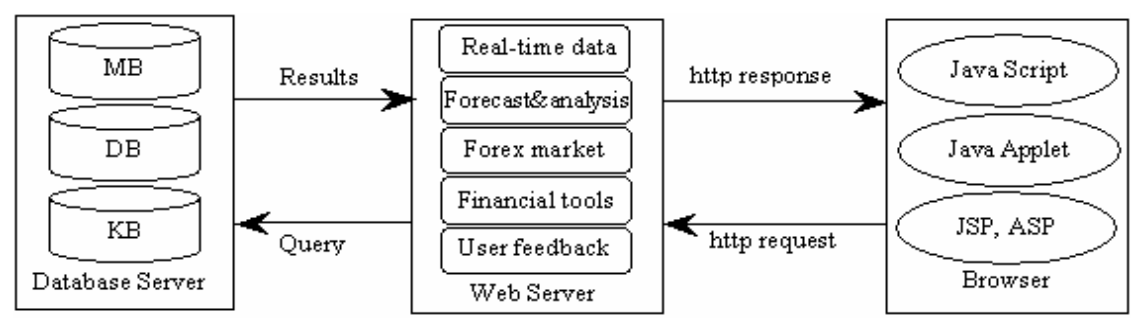

Fig. 5. Three-tier structure of WFTDSS

For explanation, WFTDSS partial user interfaces are shown in Fig. 6. Fig. 6(a) shows the interface of the trading decision criterion selection, which presents four types of decision criteria alternatives. Of the four, only one is selected by users every time. As shown in Fig. 6 (b), the interface of the fourth trading criterion mentioned previously is presented. In the interface, the corresponding optimal trading strategies will be generated by the WFTDSS as long as users or decision-makers can input certain real values or select certain "option buttons". In the same way, WFTDSS is also used as an individual system.

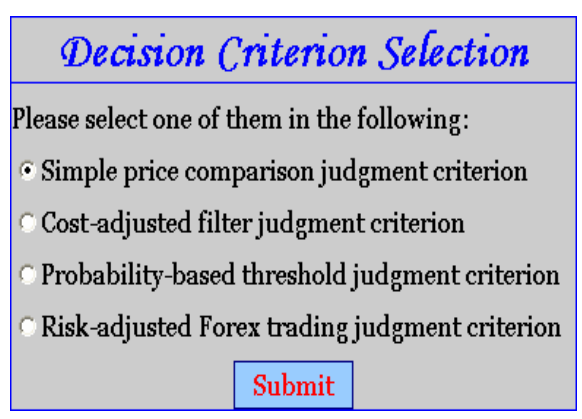

(a)

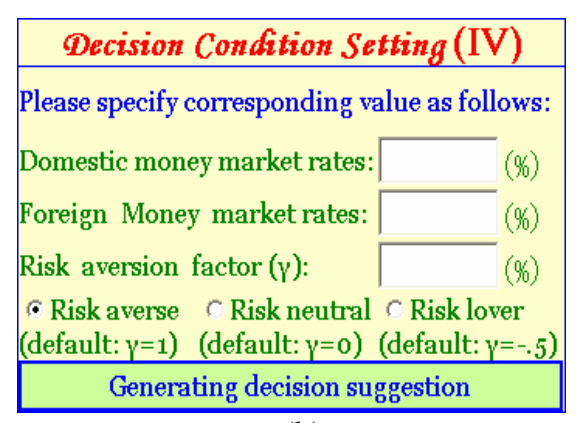

(b)

Fig. 6. WFTDSS partial user interface

\section{The Formation of IFRFTDSS}

Based on the general integrated framework and the description of two subsystems previously, we construct an integrated forex rolling forecasting and trading decision support system (IFRFTDSS) that incorporates BPNNFRFS and WFTDSS for users' convenience. Generally, the BPNNFRFS provides forex forecasting data; the WFTDSS provides trading rules and trading decisions using prediction results that are presented by the BPNNFRFS. As a whole, the formation of IFRFTDSS is the integration of the BPNNFRFS and WFTDSS. The integration process is that the BPNNFRFS is embedded into the WFTDSS. 
The BPNNFRFS is a forex forecasting system that is developed by Matlab software with the use of the neural network toolbox. This system adopts the client/server $(\mathrm{C} / \mathrm{S})$ mode here. The advantage of this mode is that the server's function can be exerted as it can in terms of either program execution efficiency or program interaction as Fig. 2 indicates, but its main disadvantage is that the client program must be installed in the every client, which lowers the holistic efficiency of the software system. In BPNNFRFS, the input data is the univariate forex time series. These sample data are transformed into appropriate input matrix vectors by corresponding rules and heuristic algorithms. Empirical results reveal that we can obtain better forecasting results than those of other statistical models [8]. Thus, the BPNN model can be used as an alternative forecasting tool for exchange rates to achieve greater forecasting accuracy and improve the prediction quality further.

In order to facilitate using the forecasting results presented by BPNNFRFS and translating the forecasting results into corresponding trading decision information, the WFTDSS is constructed. The WFTDSS can be seen as an extension of BPNNFRFS to some extent. In the same way, the BPNNFRFS is considered to be an embedded system and has been a part of WFTDSS in this integrated framework. Based on some models and knowledge bases and databases, we develop WFTDSS with a three-tier structure adopting popular web technology and using the popular browser/server mode. The advantage of this mode lies in easy operating and easy maintenance because all programs are installed in the server site. Moreover, programs can be edited and revised remotely in the client site if access is authorized. Its disadvantage is that the program execution efficiency will be low when a large number of computations exist.

Based on BPNNFRFS and WFTDSS with their three-tier structure, the IFRFTDSS is generated naturally in order to overcome the drawbacks of the two subsystems. BPNNFRFS is seen as an embedded system and has already been combined with WFTDSS in the integrated system. In the IFRFTDSS, the operations are as follows. When entering the WFTDSS, we can click the "BPNNFRFS" button; then the BPNNFRFS will run in the server site according to the corresponding program design, and meanwhile the forecasting results obtained will be transmitted into the corresponding DB of the database server. Fig. 7 shows an IFRFTDSS interface when the first trading judgment criterion is selected by users.

By implementation of IFRFTDSS and practical application, we find that the DSS is integrated, user-oriented, the forecasting performance is high and thus their decision suggestions are reliable by practical testing.

\section{Conclusions}

This study briefly introduces an integrated framework for forex rolling forecasting and trading decision, and implementation of the framework. Based on the proposed framework, we construct an integrated forex rolling forecasting and trading decision support system (IFRFTDSS), which not only incorporates BPNNFRFS and WFTDSS, but also improves the overall performance of forecasting and decision process as well as providing a more organized and user-friendly interface specifically oriented for decision making in forex market investments. 


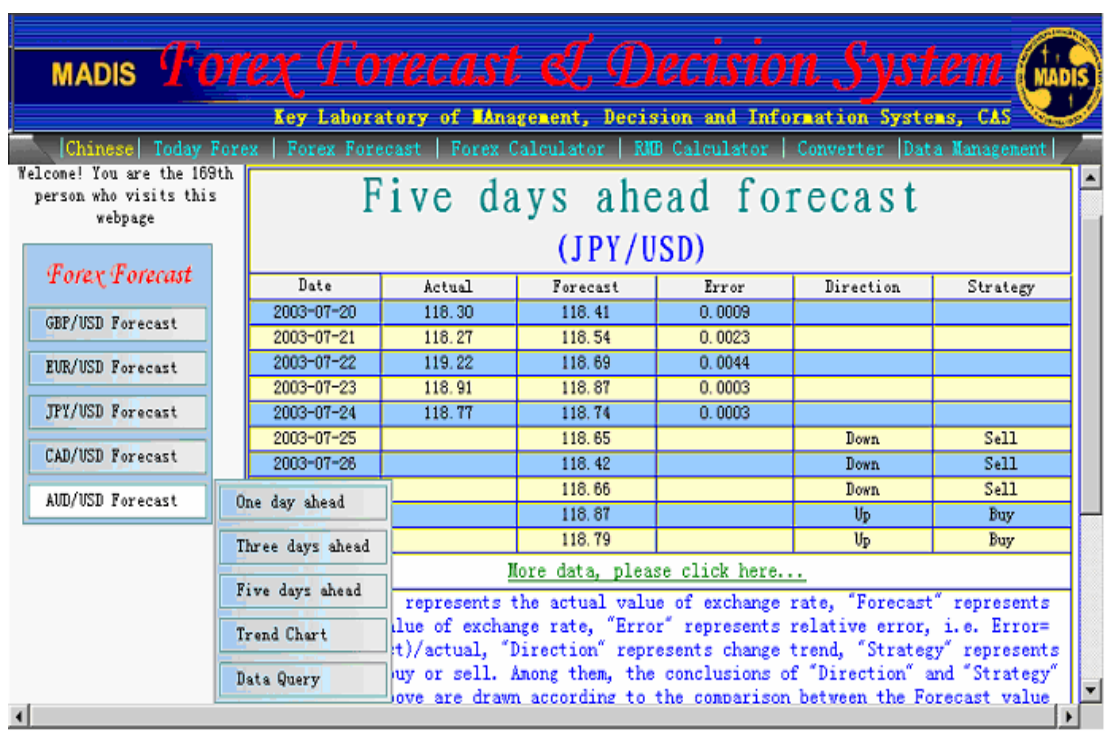

Fig. 7. The implementation of IFRFTDSS

IFRFTDSS is based on our proposed framework, which supports the flexible and interactive approach in system design and implementation, since users can visit the IFRFTDSS website and obtain real-time information and trading recommendations to adjust their own investment strategies. This system provides ease of use as well as a fast response time, which we believe is critical to all users, especially executive decision makers. At the same time, this system also provides a multidimensional data view and graphic display to help aid users' analysis. Furthermore, practical applications of the integrated system reveal that forecasting accuracy of this integrated system is very high and its trading recommendations are reliable.

\section{Acknowledgements}

The work described in this paper was fully support by grants from the Chinese Academy of Sciences and City University of Hong Kong (Project No. 7001677).

\section{References}

[1] Quah, T.S., Tan, C.L., Raman, K.S., Srinivasan, B.: Towards integrating rule-based expert systems and neural networks. Decision Support Systems, 17 (1996) 99-118

[2] Kean, P.G.W., Scott Morton, M.S.: Decision Support Systems: An Organizational Perspective. Addison- Wesley, Reading (1978)

[3] Tsoi, A.C., Tan, C.N.W., Lawrence S.: Financial time series forecasting: Application of artificial neural network techniques. Working paper (1993) 
[4] Faraway, J., Chatfield, C.: Time series forecasting with neural networks: a comparative study using the airline data. Applied Statistics 47 (2) (1998) 231-250

[5] Kaastra, I., Boyd, M.S.: Forecasting futures trading volume using neural networks. The Journal of Futures Markets 15 (8), (1995) 953-970

[6] Freeman, J.A., Skapura, D.M.: Neural Networks: Algorithms, Applications and Programming Techniques. Addison-Wesley, MA (1991)

[7] Maier, H.R., Dandy, G.C.: Neural networks for the prediction and forecasting of water resources variables: a review of modeling issues and applications. Environmental Modelling \& Software, 15 (2000) 101-124

[8] Yu, L.A., Wang, S.Y., Lai, K. K.: Exchange rates rolling forecasting using neural network model with parameter adaptive control. In: S. Chen et al. (Eds), Financial Systems Engineering, Global-Link Publishers, Hong Kong, (2003) 330-346

[9] Chen, A.S., Leung, M.T.: Regression network for error correction in foreign exchange forecasting and trading. Computers \& Operations Research, 31(7) (2004) 1049-1068 